\title{
Contributions of Spherical Harmonics to Gravitational Moment
}

\author{
Carlos M. Roithmayr* \\ NASA Langley Research Center, Hampton, Virginia, 23681
}

\begin{abstract}
A scalar gravitational potential function expressed as a series of spherical harmonics frequently serves as the basis for a model of an astronomical body's gravitational field. The contribution of a generic spherical harmonic to gravitational gradient is expressed as a dyadic, which is then used to obtain an analytical expression in vector-dyadic form for the contribution to the moment of gravitational forces about the mass center of a small body such as a spacecraft. The expression developed for a harmonic's contribution to gravitational gradient can be applied in areas beyond the scope of the paper; for example, gravitational gradient plays an important role in the state propagation matrix and the state transition matrix that are used in spacecraft trajectory targeting and Kalman filtering. Additionally, it can be employed in numerical simulations of orbit determination based on measurements obtained with a gradiometer in low-Earth orbit. Contributions of spherical harmonics to gravitational moment may be of interest in connection with attitude control of a spacecraft in the vicinity of a body with an irregular shape, such as an asteroid. Normalized spherical harmonic coefficients up to degree and order 10 are obtained for the asteroid 216 Kleopatra and used in numerical evaluations of contributions to gravitational moment.
\end{abstract}

\section{Introduction}

SPHERICAL harmonics are widely used to characterize the magnetic and gravitational fields of Earth, as Nwell as the gravitational fields of irregularly shaped bodies such as asteroids, comets, and the moons of Mars. A sphere that circumscribes every material point of an astronomical body is referred to as a Brillouin sphere; convergence of a spherical harmonic series is guaranteed in the region of space outside of this sphere, but convergence is not guaranteed inside. In the case of Earth, the radius of the Brillouin sphere is equal to the mean equatorial radius, $6,378 \mathrm{~km}$. For example, points above the North or South pole at altitudes less than $20 \mathrm{~km}$ are inside the Brillouin sphere. A polyhedral gravity model (Ref. [1]) is valid down to the surface of the polyhedron; however, it is significantly more computationally expensive than a spherical harmonic gravitational model. A polyhedral model is employed for bodies with irregular shapes at distances near or below the radius of the Brillouin sphere.

A scalar gravitational potential function expressed as a series of spherical harmonics forms the basis for a gravitational model. The first derivative of the potential with respect to position vector is the gravitational force per unit mass, which is a vector. The second derivative of the potential with respect to position is a dyadic, known as the gravitational gradient, and it can be used to construct the moment of gravitational forces about the mass center of a small body such as a spacecraft. This moment is sometimes referred to as gravity-gradient torque. Each spherical harmonic makes a contribution to gravitational moment. These contributions are typically neglected for spacecraft in Earth orbit, but they may be of interest when considering attitude behavior in the vicinity of an asteroid with highly irregular shape or mass distribution.

Zonal harmonics are independent of longitude, sectoral harmonics are independent of latitude, and tesseral harmonics are dependent on latitude and longitude. Expressions for contributions to gravitational moment provided in Refs. [2] and [3] are limited to the zonal harmonics. The purpose of this paper is to extend the work in those publications to deal with sectoral and tesseral harmonics.

The expressions related to gravitational gradient provided in the paper are useful for purposes other than construction of gravitational moment. In studies of spacecraft orbital motion it is often advantageous to

*Senior Aerospace Engineer, Vehicle Analysis Branch, MS 451, Senior Member. 
work with a state deviation, a difference between the state (position and velocity) on an actual trajectory and the state on a reference trajectory. In linearized first-order differential equations that govern the state deviation, a part of the state propagation matrix involves gravitational gradient. The state propagation matrix plays a prominent part in the first-order differential equations that govern the state transition matrix (Refs. [4] and [5]). Trajectory targeting and Kalman filtering both rely on the state transition matrix. Recently, researchers have proposed autonomous low-Earth orbit determination using a Kalman filter to process measurements of gravitational gradient obtained from a gradiometer (Refs. [6] and [7]). Expressions presented here in connection with the contribution of a spherical harmonic to gravitational gradient can be used in numerical simulations involving gradiometer measurements obtained in low-Earth orbit.

The remainder of the paper is organized as follows. Section II begins with a review of the gravitational potential expressed as a spherical harmonic series. The section continues and concludes, respectively, with expressions for the contribution of a generic spherical harmonic to gravitational force per unit mass and to the gravitational gradient dyadic. The contribution of a spherical harmonic to gravitational moment is presented in vector-dyadic form in Sec. III, and a few useful recursion relations are reviewed in Sec. IV. The validity of the expressions for contributions to gravitational force per unit mass and gravitational gradient is established in Sec. V. Spherical harmonic coefficients up to degree and order 10 are obtained for the asteroid 216 Kleopatra and used in numerical evaluations of gravitational moment contributions in Sec. VI.

\section{Gravitational Potential, and Derivatives with Respect to Position}

Following a brief review of spherical harmonic contributions to gravitational potential and gravitational force per unit mass, an expression is set forth for the contribution to gravitational gradient.

\section{A. Gravitational Potential}

The gravitational potential $V$ of a body $E$ can be expressed as an infinite series of spherical harmonics,

$$
V=\frac{\mu}{R}\left[1+\sum_{n=1}^{\infty} \sum_{m=0}^{n}\left(\frac{R_{E}}{R}\right)^{n} P_{n, m}\left(\mathrm{~s}_{\lambda}\right)\left(C_{n, m} \cos m \phi+S_{n, m} \sin m \phi\right)\right]
$$

where $\mu$, the gravitational parameter, is the product of $G$, the universal gravitational constant, and $M$, the mass of $E$. The position vector from a point $O$ fixed in $E$ to a generic point $Q$ is represented by $\mathbf{R}$ and has magnitude $R . R_{E}$ is a scaling radius for $E . P_{n, m}$ is the associated Legendre function of the first kind, of degree $n$ and order $m$, and has as its argument $\mathrm{s}_{\lambda}$, which denotes the sine of $\lambda$, the latitude of $Q$. The longitude of $Q$ is denoted by $\phi . C_{n, m}$ and $S_{n, m}$ are unnormalized gravitational coefficients of degree $n$ and order $m$. If point $O$ is chosen to be coincident with the mass center of $E$, then $C_{1,0}, C_{1,1}$, and $S_{1,1}$ all vanish as discussed, for example, in Refs. [8] and [9].

Equation (1) can be expressed in the following compact form

$$
V=\frac{\mu}{R}+\sum_{n=1}^{\infty} \sum_{m=0}^{n} V_{n, m}
$$

when $V_{n, m}$ is defined by Eq. (2) of Ref. [10],

$$
V_{n, m}=\frac{\mu R_{E}^{n}}{R^{n+m+1}} A_{n, m}\left(C_{n, m} \mathcal{C}_{m}+S_{n, m} \mathcal{S}_{m}\right)
$$

where $A_{n, m}$, referred to as a derived Legendre polynomial of degree $n$ and order $m$, is related to the associated Legendre function $P_{n, m}$ by Eq. (9b) of Ref. [11] (see also p. 3 of Ref. [10]).

$$
P_{n, m}\left(\mathrm{~s}_{\lambda}\right)=(\cos \lambda)^{m} A_{n, m}\left(\mathrm{~s}_{\lambda}\right)=\left(\frac{\rho}{R}\right)^{m} A_{n, m}\left(\mathrm{~s}_{\lambda}\right)
$$

Let $\hat{\mathbf{e}}_{1}, \hat{\mathbf{e}}_{2}$, and $\hat{\mathbf{e}}_{3}$ be a set of dextral, mutually perpendicular unit vectors fixed in $E$. $\hat{\mathbf{e}}_{1}$ and $\hat{\mathbf{e}}_{2}$ are typically regarded as fixed in the equatorial plane of $E$, with longitude measured from $\hat{\mathbf{e}}_{1}$. $\hat{\mathbf{e}}_{3}$ is used to determine the latitude of $Q$; that is, $\mathbf{s}_{\lambda}=\hat{\mathbf{r}} \cdot \hat{\mathbf{e}}_{3}$, where unit vector $\hat{\mathbf{r}}$ has the same direction as $\mathbf{R}$. The quantity $\rho$ is related to $\mathbf{R}$ by the definition

$$
\rho^{2} \triangleq\left(\mathbf{R} \cdot \hat{\mathbf{e}}_{1}\right)^{2}+\left(\mathbf{R} \cdot \hat{\mathbf{e}}_{2}\right)^{2}
$$


In words, $\rho$ is the magnitude of the projection of $\mathbf{R}$ onto the equatorial plane. The quantities $\mathcal{S}_{m}$ and $\mathcal{C}_{m}$ introduced in Eq. (3) are defined on p. 4 of Ref. [10] as

$$
\mathcal{S}_{m} \triangleq \rho^{m} \sin m \phi \quad \mathcal{C}_{m} \triangleq \rho^{m} \cos m \phi
$$

\section{B. Gravitational Force per Unit Mass}

The gravitational force per unit mass $\mathbf{f}$ exerted by $E$ at point $Q$, a vector, is obtained by differentiating the scalar potential with respect to the position vector $\mathbf{R}$ from $O$ to $Q$. That is,

$$
\mathbf{f}=\nabla V=\nabla \frac{\mu}{R}+\sum_{n=1}^{\infty} \sum_{m=0}^{n} \nabla V_{n, m}
$$

where $\nabla$ denotes differentiation with respect to the position vector $\mathbf{R}$. The result of carrying out the differentiation is shown in Ref. [12] to be

$$
\mathbf{f}=-\frac{\mu}{R^{2}} \hat{\mathbf{r}}+\sum_{n=1}^{\infty} \sum_{m=0}^{n} \mathbf{f}_{n, m}
$$

where $\mathbf{f}_{n, m}$ represents the contribution to $\mathbf{f}$ of the spherical harmonic of degree $n$ and order $m$, given by

$$
\begin{aligned}
\mathbf{f}_{n, m}= & \frac{\mu R_{E}{ }^{n}}{R^{n+m+1}}\left\{\frac{C_{n, m} \mathcal{C}_{m}+S_{n, m} \mathcal{S}_{m}}{R}\left[A_{n, m+1} \hat{\mathbf{e}}_{3}-\left(\mathrm{s}_{\lambda} A_{n, m+1}+(n+m+1) A_{n, m}\right) \hat{\mathbf{r}}\right]\right. \\
& \left.+m A_{n, m}\left[\left(C_{n, m} \mathcal{C}_{m-1}+S_{n, m} \mathcal{S}_{m-1}\right) \hat{\mathbf{e}}_{1}+\left(S_{n, m} \mathcal{C}_{m-1}-C_{n, m} \mathcal{S}_{m-1}\right) \hat{\mathbf{e}}_{2}\right]\right\}
\end{aligned}
$$

\section{Gradient of Gravitational Force per Unit Mass}

The second derivative of $V$ with respect to $\mathbf{R}$, which is the first derivative of $\mathbf{f}$ with respect to $\mathbf{R}$, is a symmetric dyadic. A dyadic $\underline{\mathbf{S}}$ is symmetric if it satisfies the relationship $\mathbf{v} \cdot \underline{\mathbf{S}}=\underline{\mathbf{S}} \cdot \mathbf{v}$ for any vector $\mathbf{v}$.

$$
\nabla \nabla V=\frac{\mu}{R^{3}}(3 \hat{\mathbf{r}} \hat{\mathbf{r}}-\underline{\mathbf{U}})+\sum_{n=1}^{\infty} \sum_{m=0}^{n} \underline{\mathbf{D}}_{n, m}
$$

where the unit dyadic $\underline{\mathbf{U}}$ is defined such that, for any vector $\mathbf{v}, \mathbf{v} \cdot \underline{\mathbf{U}}=\underline{\mathbf{U}} \cdot \mathbf{v}=\mathbf{v}$. The dyadic $\underline{\mathbf{D}}_{n, m}$ is symmetric and is given by

$$
\begin{aligned}
\underline{\mathbf{D}}_{n, m}= & \nabla \nabla V_{n, m} \\
= & \mu R_{E}{ }^{n}\left\{\Gamma_{1} \hat{\mathbf{r}} \hat{\mathbf{r}}+\Gamma_{2} \hat{\mathbf{e}}_{3} \hat{\mathbf{e}}_{3}+\Gamma_{3}\left(\hat{\mathbf{e}}_{3} \hat{\mathbf{r}}+\hat{\mathbf{r}} \hat{\mathbf{e}}_{3}\right)\right. \\
& +\Gamma_{4}\left(\hat{\mathbf{e}}_{1} \hat{\mathbf{e}}_{3}+\hat{\mathbf{e}}_{3} \hat{\mathbf{e}}_{1}\right)+\Gamma_{5}\left(\hat{\mathbf{e}}_{2} \hat{\mathbf{e}}_{3}+\hat{\mathbf{e}}_{3} \hat{\mathbf{e}}_{2}\right) \\
& +\Gamma_{6}\left(\hat{\mathbf{e}}_{1} \hat{\mathbf{r}}+\hat{\mathbf{r}} \hat{\mathbf{e}}_{1}\right)+\Gamma_{7}\left(\hat{\mathbf{e}}_{2} \hat{\mathbf{r}}+\hat{\mathbf{r}} \hat{\mathbf{e}}_{2}\right) \\
& \left.+\Gamma_{8}\left(\hat{\mathbf{e}}_{1} \hat{\mathbf{e}}_{1}-\hat{\mathbf{e}}_{2} \hat{\mathbf{e}}_{2}\right)+\Gamma_{9}\left(\hat{\mathbf{e}}_{1} \hat{\mathbf{e}}_{2}+\hat{\mathbf{e}}_{2} \hat{\mathbf{e}}_{1}\right)+\Gamma_{10} \underline{\mathbf{U}}\right\}
\end{aligned}
$$

where the coefficients $\Gamma_{1}, \ldots, \Gamma_{10}$ are defined as follows.

$$
\begin{aligned}
\Gamma_{1} \triangleq & \frac{\left(C_{n, m} \mathcal{C}_{m}+S_{n, m} \mathcal{S}_{m}\right)}{R^{n+m+3}}\left\{\mathrm{~s}_{\lambda}\left[\mathrm{s}_{\lambda} A_{n, m+2}+(2 n+2 m+5) A_{n, m+1}\right]\right. \\
& \left.+(n+m+1)(n+m+3) A_{n, m}\right\} \\
\Gamma_{2} \triangleq & \frac{A_{n, m+2}}{R^{n+m+3}}\left(C_{n, m} \mathcal{C}_{m}+S_{n, m} \mathcal{S}_{m}\right) \\
\Gamma_{3} \triangleq & \frac{-\left(C_{n, m} \mathcal{C}_{m}+S_{n, m} \mathcal{S}_{m}\right)}{R^{n+m+3}}\left[\mathrm{~s}_{\lambda} A_{n, m+2}+(n+m+2) A_{n, m+1}\right] \\
\Gamma_{4} \triangleq & \frac{m A_{n, m+1}}{R^{n+m+2}}\left(C_{n, m} \mathcal{C}_{m-1}+S_{n, m} \mathcal{S}_{m-1}\right)
\end{aligned}
$$




$$
\begin{aligned}
\Gamma_{5} & \triangleq \frac{m A_{n, m+1}}{R^{n+m+2}}\left(S_{n, m} \mathcal{C}_{m-1}-C_{n, m} \mathcal{S}_{m-1}\right) \\
\Gamma_{6} & \triangleq \frac{-A_{n, m}}{R^{n+m+2}} m(n+m+1)\left(C_{n, m} \mathcal{C}_{m-1}+S_{n, m} \mathcal{S}_{m-1}\right)-\mathrm{s}_{\lambda} \Gamma_{4} \\
\Gamma_{7} & \triangleq \frac{-A_{n, m}}{R^{n+m+2}} m(n+m+1)\left(S_{n, m} \mathcal{C}_{m-1}-C_{n, m} \mathcal{S}_{m-1}\right)-\mathrm{s}_{\lambda} \Gamma_{5} \\
\Gamma_{8} & \triangleq \frac{A_{n, m}}{R^{n+m+1}} m(m-1)\left(C_{n, m} \mathcal{C}_{m-2}+S_{n, m} \mathcal{S}_{m-2}\right) \\
\Gamma_{9} & \triangleq \frac{A_{n, m}}{R^{n+m+1}} m(m-1)\left(S_{n, m} \mathcal{C}_{m-2}-C_{n, m} \mathcal{S}_{m-2}\right) \\
\Gamma_{10} & \triangleq \frac{-\left(C_{n, m} \mathcal{C}_{m}+S_{n, m} \mathcal{S}_{m}\right)}{R^{n+m+3}}\left[\mathrm{~s}_{\lambda} A_{n, m+1}+(n+m+1) A_{n, m}\right]
\end{aligned}
$$

It can be shown that Eqs. (10)-(21) are in agreement with the expression presented in matrix form in Eq. (5-9) of Ref. [13]. It is worth noting that the dyads $\hat{\mathbf{e}}_{1} \hat{\mathbf{e}}_{1}, \hat{\mathbf{e}}_{2} \hat{\mathbf{e}}_{2}, \hat{\mathbf{e}}_{3} \hat{\mathbf{e}}_{3}$ and the dyadics $\hat{\mathbf{e}}_{1} \hat{\mathbf{e}}_{2}+\hat{\mathbf{e}}_{2} \hat{\mathbf{e}}_{1}$, $\hat{\mathbf{e}}_{1} \hat{\mathbf{e}}_{3}+\hat{\mathbf{e}}_{3} \hat{\mathbf{e}}_{1}, \hat{\mathbf{e}}_{2} \hat{\mathbf{e}}_{3}+\hat{\mathbf{e}}_{3} \hat{\mathbf{e}}_{2}$, and $\underline{\mathbf{U}}$ appearing in Eqs. (10) and (11) are independent of $\mathbf{R}$. Moreover, the coefficients $\Gamma_{4}, \ldots, \Gamma_{7}$ need not be computed when $m=0$, and $\Gamma_{8}$ and $\Gamma_{9}$ vanish when $m=0$ or $m=1$.

\section{Gravitational Moment}

A rigid body $B$ can be regarded as small in comparison to $E$ when the distance $R$ between $O$ and $B^{\star}$, the mass center of $B$, exceeds the greatest distance from $B^{\star}$ to any point of $B$. In that case, the moment $\mathbf{M}$ about $B^{\star}$ of the gravitational forces exerted by $E$ on $B$ is given approximately by Eq. (2.18.1) of Ref. [14],

$$
\mathbf{M}=-\underline{\mathbf{I}}^{\times} \cdot \nabla \nabla V
$$

where $\underline{\mathbf{I}}$ is the central inertia dyadic of $B$, and ${ }^{\times}$. denotes the cross-dot product of the two dyadics, $\underline{\mathbf{I}}$ and $\nabla \nabla V$. Here, the point $Q$ is taken to be $B^{\star}$, so that $\mathbf{R}$ is specifically the position vector from $O$ to $B^{\star}$. Two useful cross-dot identities are established in Eqs. (19) and (20) of Ref. [2]. First, $\underline{\mathbf{I}}^{\times} \cdot \underline{\mathbf{U}}=\mathbf{0}$, where $\mathbf{0}$ is the zero vector. Second, for any dyad $\mathbf{u v}$ made up of vectors $\mathbf{u}$ and $\mathbf{v},-\underline{\mathbf{I}} \times \mathbf{x} \mathbf{u v}=\mathbf{u} \times \underline{\mathbf{I}} \cdot \mathbf{v}$. Hence, substitution from Eqs. (10) and (11) into (22) leads directly to

$$
\mathbf{M}=\frac{3 \mu}{R^{3}} \hat{\mathbf{r}} \times \underline{\mathbf{I}} \cdot \hat{\mathbf{r}}+\sum_{n=1}^{\infty} \sum_{m=0}^{n} \mathbf{M}_{n, m}
$$

where $\mathbf{M}_{n, m}$, the contribution to the gravitational moment of the spherical harmonic of degree $n$ and order $m$, is given by

$$
\begin{aligned}
\mathbf{M}_{n, m} & =\mu R_{E}^{n}\left\{\Gamma_{1} \hat{\mathbf{r}} \times \underline{\mathbf{I}} \cdot \hat{\mathbf{r}}+\Gamma_{2} \hat{\mathbf{e}}_{3} \times \underline{\mathbf{I}} \cdot \hat{\mathbf{e}}_{3}+\Gamma_{3}\left(\hat{\mathbf{e}}_{3} \times \underline{\mathbf{I}} \cdot \hat{\mathbf{r}}+\hat{\mathbf{r}} \times \underline{\mathbf{I}} \cdot \hat{\mathbf{e}}_{3}\right)\right. \\
& +\Gamma_{4}\left(\hat{\mathbf{e}}_{1} \times \underline{\mathbf{I}} \cdot \hat{\mathbf{e}}_{3}+\hat{\mathbf{e}}_{3} \times \underline{\mathbf{I}} \cdot \hat{\mathbf{e}}_{1}\right)+\Gamma_{5}\left(\hat{\mathbf{e}}_{2} \times \underline{\mathbf{I}} \cdot \hat{\mathbf{e}}_{3}+\hat{\mathbf{e}}_{3} \times \underline{\mathbf{I}} \cdot \hat{\mathbf{e}}_{2}\right) \\
& +\Gamma_{6}\left(\hat{\mathbf{e}}_{1} \times \underline{\mathbf{I}} \cdot \hat{\mathbf{r}}+\hat{\mathbf{r}} \times \underline{\mathbf{I}} \cdot \hat{\mathbf{e}}_{1}\right)+\Gamma_{7}\left(\hat{\mathbf{e}}_{2} \times \underline{\mathbf{I}} \cdot \hat{\mathbf{r}}+\hat{\mathbf{r}} \times \underline{\mathbf{I}} \cdot \hat{\mathbf{e}}_{2}\right) \\
& \left.+\Gamma_{8}\left(\hat{\mathbf{e}}_{1} \times \underline{\mathbf{I}} \cdot \hat{\mathbf{e}}_{1}-\hat{\mathbf{e}}_{2} \times \underline{\mathbf{I}} \cdot \hat{\mathbf{e}}_{2}\right)+\Gamma_{9}\left(\hat{\mathbf{e}}_{1} \times \underline{\mathbf{I}} \cdot \hat{\mathbf{e}}_{2}+\hat{\mathbf{e}}_{2} \times \underline{\mathbf{I}} \cdot \hat{\mathbf{e}}_{1}\right)\right\}
\end{aligned}
$$

It can be shown that $\mathbf{M}_{2,0}$ is in agreement with Eq. (21) in Ref. [2], and $\mathbf{M}_{3,0}$ is the same as $\mathbf{M}_{3}$ reported in Ref. [3].

\section{Recursion Relations}

Table 1 of Ref. [11] contains recursion relation number I for $A_{n, m}$, which is numerically stable. For $n \geq 2$ and $0 \leq m \leq n-1$,

$$
A_{n, m}(u)=\frac{1}{n-m}\left[(2 n-1) u A_{n-1, m}(u)-(n+m-1) A_{n-2, m}(u)\right]
$$

where $u$ denotes the argument of $A_{n, m}$. Equations (14), (22), and (23) of Ref. [15] furnish values of derived Legendre polynomials for initiating the recursion process.

$$
A_{0,0}=1
$$




$$
\begin{gathered}
A_{n, n}(u)=(1)(3)(5) \cdots(2 n-1) \quad n \geq 1 \\
A_{n, n-1}(u)=u A_{n, n} \quad n \geq 1 \\
A_{n, m}=0 \quad m>n
\end{gathered}
$$

Setting $m=1$ in Eqs. (6), one obtains

$$
\mathcal{S}_{1}=\rho \sin \phi=\mathbf{R} \cdot \hat{\mathbf{e}}_{2} \quad \mathcal{C}_{1}=\rho \cos \phi=\mathbf{R} \cdot \hat{\mathbf{e}}_{1}
$$

These relationships provide seed values for the recursion formulae provided in Ref. [10]. For $m \geq 2$,

$$
\mathcal{S}_{m}=\mathcal{S}_{1} \mathcal{C}_{m-1}+\mathcal{C}_{1} \mathcal{S}_{m-1} \quad \mathcal{C}_{m}=\mathcal{C}_{1} \mathcal{C}_{m-1}-\mathcal{S}_{1} \mathcal{S}_{m-1}
$$

\section{Derivations}

Differentiation with respect to a vector is discussed at length in Sec. 2.9 of Ref. [14]. Two relationships reported therein will prove useful in what follows:

$$
\nabla \mathbf{R}=\underline{\mathbf{U}}
$$

and

$$
\nabla \hat{\mathbf{r}}=\frac{(\underline{\mathbf{U}}-\hat{\mathbf{r}} \hat{\mathbf{r}})}{R}
$$

where $\mathbf{R}$ is any vector whatsoever, $\hat{\mathbf{r}}$ is a unit vector having the same direction as $\mathbf{R}$, and $\nabla$ denotes differentiation with respect to $\mathbf{R}$.

If $s$ is a scalar function of $\mathbf{R}$, and $\mathbf{a}$ and $\mathbf{b}$ are any two vector functions of $\mathbf{R}$, then the material in Sec. 2.9 of Ref. [14] can be used to establish the following two rules that govern differentiation with respect to $\mathbf{R}$ of products involving $s, \mathbf{a}$, and $\mathbf{b}$.

$$
\begin{aligned}
\nabla(s \mathbf{a}) & =(\nabla s) \mathbf{a}+s(\nabla \mathbf{a}) \\
\nabla(\mathbf{a} \cdot \mathbf{b}) & =(\nabla \mathbf{a}) \cdot \mathbf{b}+(\nabla \mathbf{b}) \cdot \mathbf{a}
\end{aligned}
$$

In general, the dyad $\mathbf{a b}$ is not symmetric; in other words, for any vector $\mathbf{v}, \mathbf{v} \cdot \mathbf{a b} \neq \mathbf{a b} \cdot \mathbf{v}$. Likewise, $\mathbf{b a}$ is unsymmetric, and is not the same dyad as ab. The first term appearing on the right hand side of Eq. (34) is a dyad; therefore, the order of the two vectors $(\nabla s)$ and $\mathbf{a}$ is important. Furthermore, the dyads $(\nabla \mathbf{a})$ and $(\nabla \mathbf{b})$ may be unsymmetric, and the orders of the dot products on the right hand side of Eq. (35) are important.

After noting that $R=(\mathbf{R} \cdot \mathbf{R})^{1 / 2}$, one may obtain another relationship that will be called upon several times in the sequel. Keeping Eq. (35) in mind, one can write

$$
\nabla \frac{1}{R^{n}}=\nabla(\mathbf{R} \cdot \mathbf{R})^{-n / 2}=-\frac{n}{2}(\mathbf{R} \cdot \mathbf{R})^{-(1+n / 2)}(\underline{\mathbf{U}} \cdot \mathbf{R}+\underline{\mathbf{U}} \cdot \mathbf{R})=-n \frac{\mathbf{R}}{R^{n+2}}
$$

Thus, when proceeding from Eq. (7) to (8), one obtains with $n=1$ the expected result for the first term on the right-hand side, $\nabla \mu / R=-\mu \mathbf{R} / R^{3}=-\mu \hat{\mathbf{r}} / R^{2}$.

For convenience, Eq. (3) can be rewritten as

$$
V_{n, m}=\mu R_{E}{ }^{n} \frac{A_{n, m} T_{n, m}}{R^{n+m+1}}
$$

where the scalar $T_{n, m}$ is defined as

$$
T_{n, m} \triangleq C_{n, m} \mathcal{C}_{m}+S_{n, m} \mathcal{S}_{m}
$$

The first derivative of $V_{n, m}$ with respect to $\mathbf{R}$ is then

$$
\nabla V_{n, m}=\mu R_{E}{ }^{n}\left[\frac{A_{n, m} \nabla T_{n, m}+T_{n, m} \nabla A_{n, m}}{R^{n+m+1}}+\left(T_{n, m} A_{n, m}\right) \nabla \frac{1}{R^{n+m+1}}\right]
$$

Equation (10) in Ref. [11] allows one to write

$$
\begin{gathered}
\nabla A_{n, m}(u)=\frac{d}{d u} A_{n, m}(u) \nabla u=A_{n, m+1}(u) \nabla u \\
5 \text { of } 15
\end{gathered}
$$


where the symbol $u$ represents the argument of $A_{n, m}$. Hence,

$$
\nabla A_{n, m}\left(\mathrm{~s}_{\lambda}\right)=A_{n, m+1}\left(\mathrm{~s}_{\lambda}\right) \nabla \mathrm{s}_{\lambda}
$$

After noting that $\hat{\mathbf{e}}_{3}$ is independent of $\mathbf{R}$, in view of Eq. (33), one can write

$$
\nabla \mathrm{s}_{\lambda}=\nabla\left(\hat{\mathbf{r}} \cdot \hat{\mathbf{e}}_{3}\right)=\frac{(\underline{\mathbf{U}}-\hat{\mathbf{r}} \hat{\mathbf{r}})}{R} \cdot \hat{\mathbf{e}}_{3}=\frac{\left(\hat{\mathbf{e}}_{3}-\hat{\mathbf{r}} \hat{\mathbf{r}} \cdot \hat{\mathbf{e}}_{3}\right)}{R}=\frac{\left(\hat{\mathbf{e}}_{3}-\mathrm{s}_{\lambda} \hat{\mathbf{r}}\right)}{R}
$$

Therefore,

$$
\nabla A_{n, m}\left(\mathrm{~s}_{\lambda}\right)=\frac{A_{n, m+1}}{R}\left(\hat{\mathbf{e}}_{3}-\mathrm{s}_{\lambda} \hat{\mathbf{r}}\right)
$$

Equations (7a) $-(7 \mathrm{~d})$ of Ref. [10] furnish an especially compact representation of the derivatives of $\mathcal{S}_{m}$ and $\mathcal{C}_{m}$ with respect to $\mathbf{R}$,

$$
\nabla \mathcal{S}_{m}=m\left(\mathcal{S}_{m-1} \hat{\mathbf{e}}_{1}+\mathcal{C}_{m-1} \hat{\mathbf{e}}_{2}\right) \quad \nabla \mathcal{C}_{m}=m\left(\mathcal{C}_{m-1} \hat{\mathbf{e}}_{1}-\mathcal{S}_{m-1} \hat{\mathbf{e}}_{2}\right)
$$

The spherical harmonic coefficients $C_{n, m}$ and $S_{n, m}$ are constants and hence independent of $\mathbf{R}$; therefore, the definition of $T_{n, m}$ in Eq. (38) can be used together with Eqs. (44) to write

$$
\nabla T_{n, m}=m\left[\left(C_{n, m} \mathcal{C}_{m-1}+S_{n, m} \mathcal{S}_{m-1}\right) \hat{\mathbf{e}}_{1}+\left(S_{n, m} \mathcal{C}_{m-1}-C_{n, m} \mathcal{S}_{m-1}\right) \hat{\mathbf{e}}_{2}\right]
$$

In view of Eq. (36), one can write

$$
\nabla \frac{1}{R^{n+m+1}}=-(n+m+1) \frac{\mathbf{R}}{R^{n+m+3}}
$$

Substitution from Eqs. (43), (45), and (46) into (39) establishes the validity of Eq. (9).

We now turn our attention to obtaining the second derivative of $V$ with respect to R. Referring to Eqs. (36) and (32), one can deal with the first term on the right-hand side of Eq. (8) by writing

$$
-\nabla \frac{\mu \hat{\mathbf{r}}}{R^{2}}=-\mu \nabla \frac{\mathbf{R}}{R^{3}}=\mu\left(\frac{3 \mathbf{R}}{R^{5}} \mathbf{R}-\frac{\underline{\mathbf{U}}}{R^{3}}\right)=\frac{\mu}{R^{3}}(3 \hat{\mathbf{r}} \hat{\mathbf{r}}-\underline{\mathbf{U}})
$$

To obtain the second derivative of $V_{n, m}$ with respect to $\mathbf{R}$, begin by rewriting Eq. (39) as

$$
\nabla V_{n, m}=\mu R_{E}^{n}\left[\frac{A_{n, m} \nabla T_{n, m}+T_{n, m} A_{n, m+1} \nabla \mathrm{s}_{\lambda}}{R^{n+m+1}}+\left(T_{n, m} A_{n, m}\right) \nabla R^{-(n+m+1)}\right]
$$

With Eq. (34) in mind, the second derivative can then be expressed as

$$
\begin{aligned}
\nabla \nabla V_{n, m}= & \mu R_{E}{ }^{n}\left\{\frac{\nabla A_{n, m} \nabla T_{n, m}+A_{n, m} \nabla \nabla T_{n, m}}{R^{n+m+1}}\right. \\
& +\frac{\left(\nabla T_{n, m} A_{n, m+1}+T_{n, m} \nabla A_{n, m+1}\right) \nabla \mathrm{s}_{\lambda}+T_{n, m} A_{n, m+1} \nabla \nabla \mathrm{s}_{\lambda}}{R^{n+m+1}} \\
& +\nabla R^{-(n+m+1)}\left(A_{n, m} \nabla T_{n, m}+T_{n, m} A_{n, m+1} \nabla \mathrm{s}_{\lambda}\right) \\
& \left.+\left(\nabla T_{n, m} A_{n, m}+T_{n, m} \nabla A_{n, m}\right) \nabla R^{-(n+m+1)}+\left(T_{n, m} A_{n, m}\right) \nabla \nabla R^{-(n+m+1)}\right\}
\end{aligned}
$$

By appealing to Eq. (41), one arrives at

$$
\begin{aligned}
\nabla \nabla V_{n, m}= & \mu R_{E}{ }^{n}\{ \\
& {\left[A_{n, m+1}\left(\frac{\nabla \nabla \mathrm{s}_{\lambda}}{R^{n+m+1}}+\nabla \mathrm{s}_{\lambda} \nabla R^{-(n+m+1)}+\nabla R^{-(n+m+1)} \nabla \mathrm{s}_{\lambda}\right)\right.} \\
+ & \left.\frac{A_{n, m+2}}{R^{n+m+1}} \nabla \mathrm{s}_{\lambda} \nabla \mathrm{s}_{\lambda}+A_{n, m} \nabla \nabla R^{-(n+m+1)}\right] T_{n, m} \\
+ & {\left[\frac{A_{n, m+1}}{R^{n+m+1}} \nabla \mathrm{s}_{\lambda}+A_{n, m} \nabla R^{-(n+m+1)}\right] \nabla T_{n, m} } \\
+ & \nabla T_{n, m}\left[\frac{A_{n, m+1}}{R^{n+m+1}} \nabla \mathrm{s}_{\lambda}+A_{n, m} \nabla R^{-(n+m+1)}\right] \\
+ & \left.\frac{A_{n, m}}{R^{n+m+1}} \nabla \nabla T_{n, m}\right\}
\end{aligned}
$$


Now, by considering Eq. (42) one may reproduce Eq. (16) in Ref. [3], which states

$$
\nabla \nabla \mathrm{s}_{\lambda}=\frac{1}{R^{2}}\left[3 \mathrm{~s}_{\lambda} \hat{\mathbf{r}} \hat{\mathbf{r}}-\left(\hat{\mathbf{e}}_{3} \hat{\mathbf{r}}+\hat{\mathbf{r}} \hat{\mathbf{e}}_{3}+\mathrm{s}_{\lambda} \underline{\mathbf{U}}\right)\right]
$$

Substitution from Eqs. (42) and (46) yields

$$
\nabla \mathrm{s}_{\lambda} \nabla R^{-(n+m+1)}+\nabla R^{-(n+m+1)} \nabla \mathrm{s}_{\lambda}=\frac{(n+m+1)}{R^{n+m+3}}\left[2 \mathrm{~s}_{\lambda} \hat{\mathbf{r}} \hat{\mathbf{r}}-\left(\hat{\mathbf{e}}_{3} \hat{\mathbf{r}}+\hat{\mathbf{r}} \hat{\mathbf{e}}_{3}\right)\right]
$$

These two relationships permit one to write the first term in the first set of square brackets on the right-hand side of Eq. (50),

$$
\begin{aligned}
A_{n, m+1} & \left(\frac{\nabla \nabla \mathrm{s}_{\lambda}}{R^{n+m+1}}+\nabla \mathrm{s}_{\lambda} \nabla R^{-(n+m+1)}+\nabla R^{-(n+m+1)} \nabla \mathrm{s}_{\lambda}\right) \\
= & \frac{A_{n, m+1}}{R^{n+m+3}}\left[3 \mathrm{~s}_{\lambda} \hat{\mathbf{r}} \hat{\mathbf{r}}-\left(\hat{\mathbf{e}}_{3} \hat{\mathbf{r}}+\hat{\mathbf{r}} \hat{\mathbf{e}}_{3}+\mathrm{s}_{\lambda} \underline{\mathbf{U}}\right)\right]+\frac{(n+m+1) A_{n, m+1}}{R^{n+m+3}}\left[2 \mathrm{~s}_{\lambda} \hat{\mathbf{r}} \hat{\mathbf{r}}-\left(\hat{\mathbf{e}}_{3} \hat{\mathbf{r}}+\hat{\mathbf{r}} \hat{\mathbf{e}}_{3}\right)\right] \\
= & \frac{A_{n, m+1}}{R^{n+m+3}}\left[(2 n+2 m+5) \mathrm{s}_{\lambda} \hat{\mathbf{r}} \hat{\mathbf{r}}-(n+m+2)\left(\hat{\mathbf{e}}_{3} \hat{\mathbf{r}}+\hat{\mathbf{r}} \hat{\mathbf{e}}_{3}\right)-\mathrm{s}_{\lambda} \underline{\mathbf{U}}\right]
\end{aligned}
$$

The right hand side of Eq. (42) can be juxtaposed with itself to yield the dyadic

$$
\nabla \mathrm{s}_{\lambda} \nabla \mathrm{s}_{\lambda}=\frac{\left(\hat{\mathbf{e}}_{3}-\mathrm{s}_{\lambda} \hat{\mathbf{r}}\right)}{R} \frac{\left(\hat{\mathbf{e}}_{3}-\mathrm{s}_{\lambda} \hat{\mathbf{r}}\right)}{R}=\frac{1}{R^{2}}\left[\mathrm{~s}_{\lambda}^{2} \hat{\mathbf{r}} \hat{\mathbf{r}}-\mathrm{s}_{\lambda}\left(\hat{\mathbf{e}}_{3} \hat{\mathbf{r}}+\hat{\mathbf{r}} \hat{\mathbf{e}}_{3}\right)+\hat{\mathbf{e}}_{3} \hat{\mathbf{e}}_{3}\right]
$$

From Eq. (46) one can obtain

$$
\nabla \nabla R^{-(n+m+1)}=(n+m+1)\left[(n+m+3) R^{-(n+m+5)} \mathbf{R R}-R^{-(n+m+3)} \underline{\mathbf{U}}\right]
$$

The terms containing the second and third sets of square brackets on the right-hand side of Eq. (50) are formed with the aid of Eqs. (42), (45), and (46); that is

$$
\begin{aligned}
& {\left[\frac{A_{n, m+1}}{R^{n+m+1}} \nabla \mathrm{s}_{\lambda}+A_{n, m} \nabla R^{-(n+m+1)}\right] \nabla T_{n, m}} \\
& =\frac{m}{R^{n+m+2}}\left[A_{n, m+1}\left(\hat{\mathbf{e}}_{3}-\mathrm{s}_{\lambda} \hat{\mathbf{r}}\right)-(n+m+1) A_{n, m} \hat{\mathbf{r}}\right]\left[\left(C_{n, m} \mathcal{C}_{m-1}+S_{n, m} \mathcal{S}_{m-1}\right) \hat{\mathbf{e}}_{1}\right. \\
& \left.\quad+\left(S_{n, m} \mathcal{C}_{m-1}-C_{n, m} \mathcal{S}_{m-1}\right) \hat{\mathbf{e}}_{2}\right]
\end{aligned}
$$

and

$$
\begin{aligned}
\nabla T_{n, m} & {\left[\frac{A_{n, m+1}}{R^{n+m+1}} \nabla \mathrm{s}_{\lambda}+A_{n, m} \nabla R^{-(n+m+1)}\right] } \\
= & \frac{m}{R^{n+m+2}}\left[\left(C_{n, m} \mathcal{C}_{m-1}+S_{n, m} \mathcal{S}_{m-1}\right) \hat{\mathbf{e}}_{1}+\left(S_{n, m} \mathcal{C}_{m-1}-C_{n, m} \mathcal{S}_{m-1}\right) \hat{\mathbf{e}}_{2}\right]\left[A_{n, m+1}\left(\hat{\mathbf{e}}_{3}-\mathrm{s}_{\lambda} \hat{\mathbf{r}}\right)\right. \\
& \left.\quad-(n+m+1) A_{n, m} \hat{\mathbf{r}}\right]
\end{aligned}
$$

Employing Eqs. (44) recursively yields

$$
\begin{aligned}
& \nabla \nabla \mathcal{S}_{m}=m(m-1)\left[\mathcal{S}_{m-2}\left(\hat{\mathbf{e}}_{1} \hat{\mathbf{e}}_{1}-\hat{\mathbf{e}}_{2} \hat{\mathbf{e}}_{2}\right)+\mathcal{C}_{m-2}\left(\hat{\mathbf{e}}_{1} \hat{\mathbf{e}}_{2}+\hat{\mathbf{e}}_{2} \hat{\mathbf{e}}_{1}\right)\right] \\
& \nabla \nabla \mathcal{C}_{m}=m(m-1)\left[\mathcal{C}_{m-2}\left(\hat{\mathbf{e}}_{1} \hat{\mathbf{e}}_{1}-\hat{\mathbf{e}}_{2} \hat{\mathbf{e}}_{2}\right)-\mathcal{S}_{m-2}\left(\hat{\mathbf{e}}_{1} \hat{\mathbf{e}}_{2}+\hat{\mathbf{e}}_{2} \hat{\mathbf{e}}_{1}\right)\right]
\end{aligned}
$$

and these relationships can be used together with Eq. (38) to obtain

$$
\begin{aligned}
\nabla \nabla T_{n, m} & =C_{n, m} \nabla \nabla \mathcal{C}_{m}+S_{n, m} \nabla \nabla \mathcal{S}_{m} \\
& =m(m-1)\left[\left(C_{n, m} \mathcal{C}_{m-2}+S_{n, m} \mathcal{S}_{m-2}\right)\left(\hat{\mathbf{e}}_{1} \hat{\mathbf{e}}_{1}-\hat{\mathbf{e}}_{2} \hat{\mathbf{e}}_{2}\right)+\left(S_{n, m} \mathcal{C}_{m-2}-C_{n, m} \mathcal{S}_{m-2}\right)\left(\hat{\mathbf{e}}_{1} \hat{\mathbf{e}}_{2}+\hat{\mathbf{e}}_{2} \hat{\mathbf{e}}_{1}\right)\right]
\end{aligned}
$$

Substitution from Eqs. (53)-(57) and (60) into (50) establishes the validity of Eq. (11). In turn, Eqs. (47) and (11) lead directly to Eq. (10). 


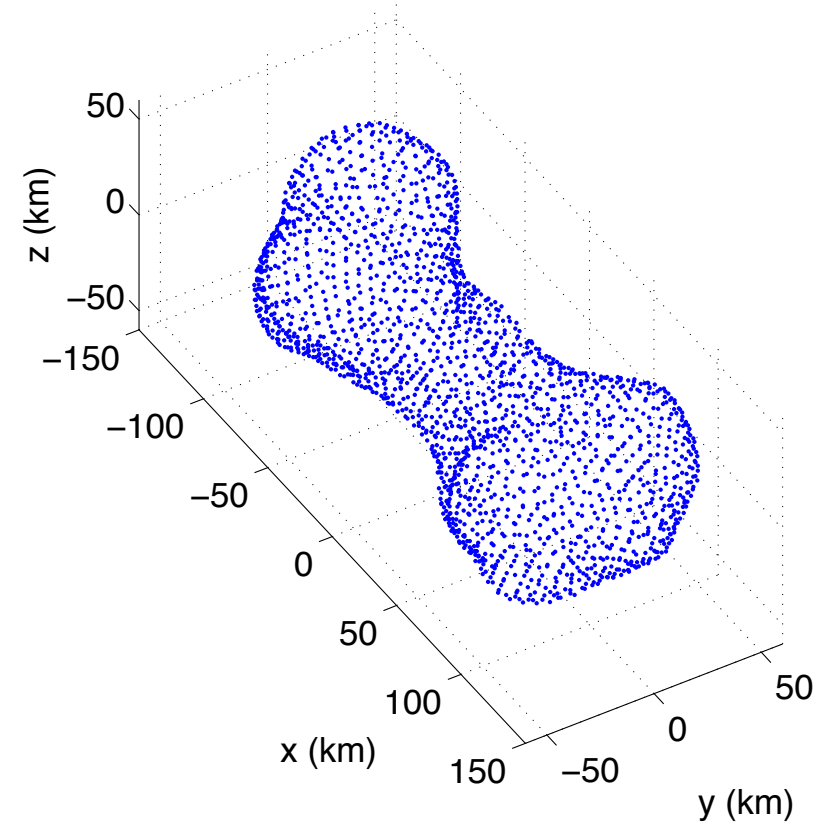

Figure 1. Kleopatra, three-dimensional view

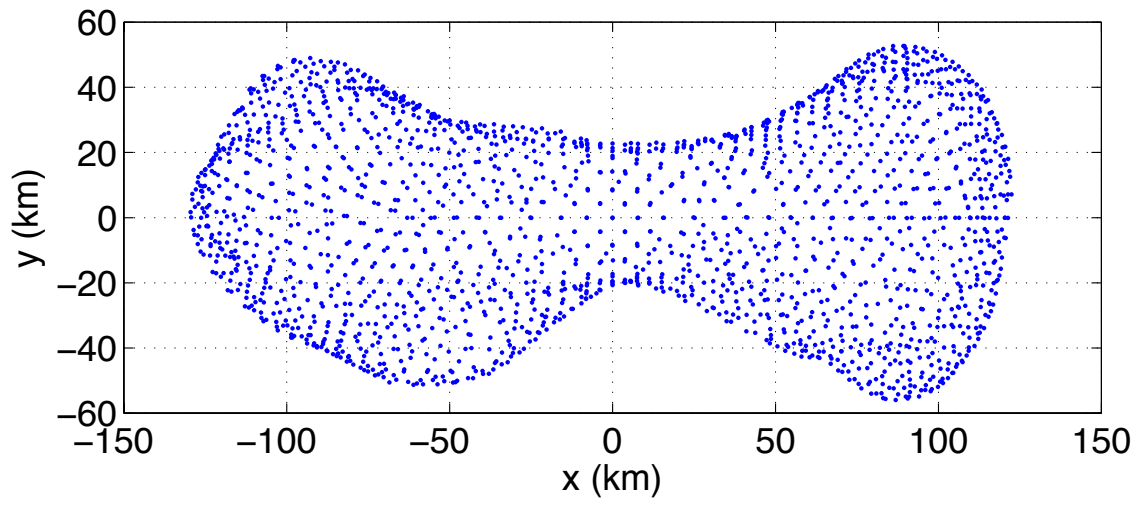

Figure 2. Kleopatra, top view

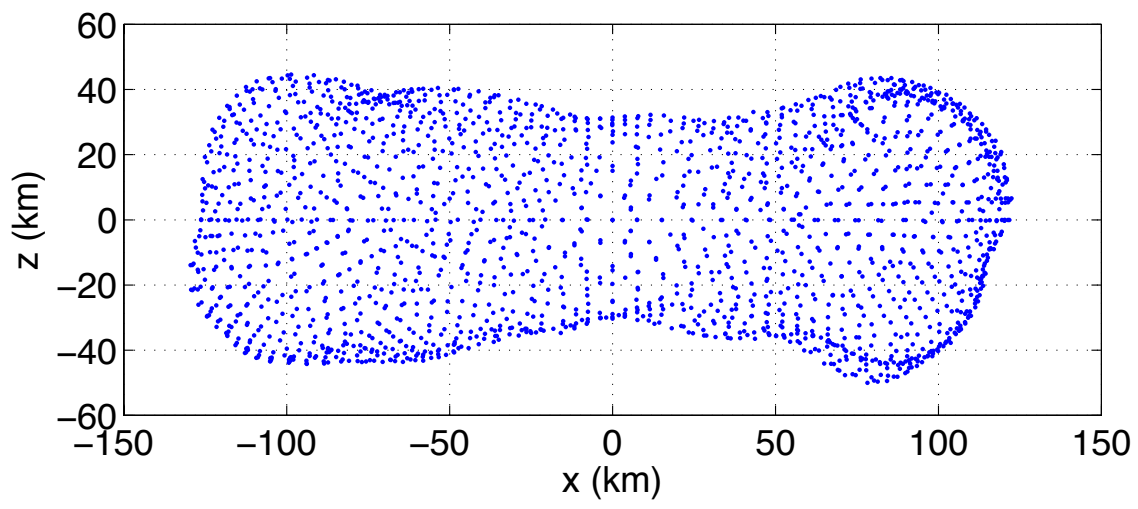

Figure 3. Kleopatra, side view 


\section{Asteroid 216 Kleopatra}

The main-belt asteroid 216 Kleopatra has a highly irregular shape; it is similar to a dog bone, as illustrated in Figs. 1-3. Thus, Kleopatra serves as a rather interesting example to employ in a numerical study of contributions of spherical harmonics to gravitational moment.

Figures 1-3 are constructed from the shape model obtained by Ostro et al. in Ref. [16]. The model is available at https://echo.jpl.nasa.gov/asteroids/shapes/kleo.obj. As discussed in Ref. [17], the size of the model provided by Ostro et al. is scaled up in order to obtain a mass of $4.64 \times 10^{18} \mathrm{~kg}$ when the density is assumed to be $4.27 \mathrm{~g} / \mathrm{cm}^{3}$. A scaling factor of 1.153032510683412 results in a volume of $1.086651053864165 \times 10^{6} \mathrm{~km}^{3}$. The original shape model is also adjusted such that the centroid becomes the reference point for the position vectors of the vertices. The maximum distance from the centroid to a vertex is $131.6 \mathrm{~km}$, which is thus the radius of the Brillouin sphere for the adjusted, scaled shape model. The approach presented in Ref. [18] is used to obtain spherical harmonic coefficients up to degree and order 10 for Kleopatra, with a scaling radius $R_{E}=143.384921778618 \mathrm{~km}$. The normalized values of the coefficients, $\bar{C}_{n, m}$ and $\bar{S}_{n, m}$, are recorded in Tables 1 and 2. A normalized coefficient is multiplied by a normalizing factor $N_{n, m}$ to obtain the corresponding unnormalized coefficient required in Eqs. (12)-(21). $N_{n, m}=\sqrt{2(2 n+1)(n-m) ! /(n+m) !}$ for $m \neq 0$, and $N_{n, m}=\sqrt{2 n+1}$ for $m=0$.

Unnormalized spherical harmonic coefficients are used together with Eq. (24) to calculate contributions to gravitational moment about the mass center of a spacecraft $B$ having a central inertia dyadic $\underline{\mathbf{I}}=I_{1} \hat{\mathbf{b}}_{1} \hat{\mathbf{b}}_{1}+$ $I_{2} \hat{\mathbf{b}}_{2} \hat{\mathbf{b}}_{2}+I_{3} \hat{\mathbf{b}}_{3} \hat{\mathbf{b}}_{3} \mathrm{~kg}-\mathrm{m}^{2}$, where $\hat{\mathbf{b}}_{1}, \hat{\mathbf{b}}_{2}$, and $\hat{\mathbf{b}}_{3}$ are dextral, mutually perpendicular unit vectors fixed in $B$ and parallel to central principal axes of inertia of $B$. The central principal moments of inertia are taken to be $I_{1}=36,070 \mathrm{~kg}-\mathrm{m}^{2}, I_{2}=98,570 \mathrm{~kg}-\mathrm{m}^{2}$, and $I_{3}=78,221 \mathrm{~kg}-\mathrm{m}^{2}$. Gravitational moment is calculated at stations that lie on a circle of radius $150 \mathrm{~km}$ inclined $45^{\circ}$ to the equatorial $(x-y)$ plane of the asteroid; the plane of the circle intersects the asteroid's equatorial plane on the asteroid's $x$ axis. The station positions are parameterized by an angle $\theta$ that has the value of 0 where the circle intersects the positive $x$ axis of Kleopatra. The orientation of $B$ is such that $\hat{\mathbf{b}}_{1}$ is always directed from the mass center of the asteroid to the mass center of $B, \hat{\mathbf{b}}_{2}$ is always tangent to the circle, and $\hat{\mathbf{b}}_{3}$ is always perpendicular to the plane of the circle. Unit vectors $\hat{\mathbf{e}}_{1}, \hat{\mathbf{e}}_{2}$, and $\hat{\mathbf{e}}_{3}$ fixed in the asteroid mark the directions of the positive $x, y$, and $z$ axes respectively. The orientation of $\hat{\mathbf{b}}_{1}, \hat{\mathbf{b}}_{2}$, and $\hat{\mathbf{b}}_{3}$ with respect to $\hat{\mathbf{e}}_{1}, \hat{\mathbf{e}}_{2}$, and $\hat{\mathbf{e}}_{3}$ can be described with the direction cosines in Table 3 , with $i=45^{\circ}$. It is easily verified that when $\hat{\mathbf{r}}$ is parallel to a central principal axis of inertia of $B, \hat{\mathbf{r}} \times \underline{\mathbf{I}} \cdot \hat{\mathbf{r}}=\mathbf{0}$. This is the case in the present example because $\hat{\mathbf{r}}=\hat{\mathbf{b}}_{1}$, and therefore the first term in each of Eqs. (23) and (24) vanishes.

Contributions of spherical harmonics of degree 2 to gravitational moment are shown in Fig. $4 . \mathbf{M}_{2,0}$, $\mathbf{M}_{2,1}$, and $\mathbf{M}_{2,2}$ are the contributions of a zonal, tesseral, and sectoral harmonic, respectively. Projections of these vectors onto $\hat{\mathbf{b}}_{1}, \hat{\mathbf{b}}_{2}$, and $\hat{\mathbf{b}}_{3}$ as functions of $\theta$ are presented in the the top, middle, and bottom plots, respectively. Equations (30)-(32) of Ref. [2] are applicable to the present example; $\mathbf{M}_{2,0}$ can be written as

$$
\mathbf{M}_{2,0}=\frac{12 \mu C_{2,0} R_{E}{ }^{2} \sin i}{R^{5}}\left[\frac{1}{4}\left(I_{3}-I_{2}\right) \cos i \cos \theta \hat{\mathbf{b}}_{1}+\left(I_{3}-I_{1}\right) \cos i \sin \theta \hat{\mathbf{b}}_{2}+\left(I_{1}-I_{2}\right) \sin i \sin \theta \cos \theta \hat{\mathbf{b}}_{3}\right]
$$

and this expression is in concert with the functional forms of the corresponding curves in Fig. 4. Although the projections of $\mathbf{M}_{2,1}$ are similar in character to those of $\mathbf{M}_{2,0}$ and $\mathbf{M}_{2,2}$, they are not evident at the scale required to display the latter contributions. It is interesting to note that $\mathbf{M}_{2,2} \cdot \hat{\mathbf{b}}_{r} \approx-\mathbf{M}_{2,0} \cdot \hat{\mathbf{b}}_{r}$ for $r=1,2$.

Projections of $\mathbf{M}_{3, m}(m=0,1,2,3)$ onto $\hat{\mathbf{b}}_{1}, \hat{\mathbf{b}}_{2}$, and $\hat{\mathbf{b}}_{3}$ are displayed in Fig. 5 . In each case the sectoral contribution $\mathbf{M}_{3,3}$ provides the dominant projection, which is an order of magnitude less than the corresponding projection of $\mathbf{M}_{2,2}$.

In practice the maximum value of the degree $n$ is set to a finite integer $N$ when gravitational force per unit mass is calculated, for example, according to Eq. (8). The calculations are then said to be associated with an $N \times N$ spherical harmonic gravity model. Calculations of gravitational moment according to Eq. (23) can be dealt with and referred to in a similar manner. Because $\hat{\mathbf{r}} \times \underline{\mathbf{I}} \cdot \hat{\mathbf{r}}=\mathbf{0}$ in the present example, $\mathbf{M}$ is given by the sum $\sum_{n=1}^{N} \sum_{m=0}^{n} \mathbf{M}_{n, m}$. Projections of $\mathbf{M}$ onto $\hat{\mathbf{b}}_{1}, \hat{\mathbf{b}}_{2}$, and $\hat{\mathbf{b}}_{3}$ are shown in Fig. 6 for $N=2,5$, and 10. As can be seen, the $10 \times 10$ gravitational moment model is poorly approximated by the $2 \times 2$ and $5 \times 5$ models for the projections onto $\hat{\mathbf{b}}_{1}$ and $\hat{\mathbf{b}}_{2}$, whereas the $5 \times 5$ model may furnish a reasonable approximation for the projection onto $\hat{\mathbf{b}}_{3}$. Contributions to gravitational moment from higher degree harmonics are thus demonstrated to be significant in the case of the asteroid Kleopatra. This result 
stands in contrast to what is observed for Earth, where $\sum_{n=2}^{3} \sum_{m=0}^{n} \mathbf{M}_{n, m}$ is virtually indistinguishable from $\mathbf{M}_{2,0}$.

Table 1. Normalized coefficients for Kleopatra, $n=0,1, \ldots, 7$

\begin{tabular}{rrrr}
\hline \hline$n$ & $m$ & $\bar{C}_{n, m}$ & $\bar{S}_{n, m}$ \\
\hline 0 & 0 & 1 & 0 \\
1 & 0 & $-9.9645 \times 10^{-18}$ & 0 \\
1 & 1 & $4.45201 \times 10^{-17}$ & $-3.12047 \times 10^{-18}$ \\
2 & 0 & -0.0563137 & 0 \\
2 & 1 & 0.000204617 & -0.000431569 \\
2 & 2 & 0.0958872 & -0.000173268 \\
3 & 0 & -0.000487783 & 0 \\
3 & 1 & -0.000224572 & -0.000481935 \\
3 & 2 & $-7.652 \times 10^{-5}$ & -0.000424934 \\
3 & 3 & -0.0018542 & 0.00291674 \\
4 & 0 & 0.00955284 & 0 \\
4 & 1 & -0.000852424 & -0.000127394 \\
4 & 2 & -0.0140889 & 0.000461535 \\
4 & 3 & 0.000838149 & 0.000418229 \\
4 & 4 & 0.0171909 & -0.00115991 \\
5 & 0 & 0.000366151 & 0 \\
5 & 1 & $-8.29736 \times 10^{-5}$ & 0.000111662 \\
5 & 2 & -0.000233617 & 0.000489825 \\
5 & 3 & 0.000604393 & -0.000523938 \\
5 & 4 & -0.000410598 & -0.000833892 \\
5 & 5 & -0.00225019 & 0.00193798 \\
6 & 0 & -0.00180719 & 0 \\
6 & 1 & 0.000599411 & 0.000107414 \\
6 & 2 & 0.00266428 & -0.000134785 \\
6 & 3 & -0.000519542 & -0.00032879 \\
6 & 4 & -0.00292046 & 0.000302123 \\
6 & 5 & 0.000395859 & 0.000530943 \\
6 & 6 & 0.00329497 & -0.00065939 \\
7 & 0 & -0.000199044 & 0 \\
7 & 1 & $-1.08159 \times 10^{-5}$ & $-1.26989 \times 10^{-5}$ \\
7 & 2 & 0.000172302 & -0.00023069 \\
7 & 3 & -0.00013192 & $7.5305 \times 10^{-5}$ \\
7 & 4 & 0.000107121 & 0.000417474 \\
7 & 5 & 0.000479654 & -0.000276905 \\
7 & 6 & -0.000398871 & -0.000495079 \\
7 & 7 & -0.00140063 & 0.000927067 \\
\hline \hline & & &
\end{tabular}

10 of 15 
Table 2. Normalized coefficients for Kleopatra, $n=8,9,10$

\begin{tabular}{rrrr}
\hline \hline$n$ & $m$ & $\bar{C}_{n, m}$ & $\bar{S}_{n, m}$ \\
\hline 8 & 0 & 0.000245673 & 0 \\
8 & 1 & -0.000329775 & $-5.14566 \times 10^{-5}$ \\
8 & 2 & -0.000394157 & $1.89922 \times 10^{-5}$ \\
8 & 3 & 0.000286091 & 0.000157709 \\
8 & 4 & 0.000510949 & $-5.08607 \times 10^{-5}$ \\
8 & 5 & -0.00021868 & -0.000267254 \\
8 & 6 & -0.000620071 & 0.000114282 \\
8 & 7 & 0.000158152 & 0.000351333 \\
8 & 8 & 0.000572136 & -0.000293814 \\
9 & 0 & $9.05715 \times 10^{-5}$ & 0 \\
9 & 1 & $7.15439 \times 10^{-5}$ & $-5.02249 \times 10^{-6}$ \\
9 & 2 & $-8.70043 \times 10^{-5}$ & $8.6136 \times 10^{-5}$ \\
9 & 3 & $-3.08506 \times 10^{-5}$ & $6.34522 \times 10^{-6}$ \\
9 & 4 & $-2.42296 \times 10^{-5}$ & -0.0001605 \\
9 & 5 & $-6.18706 \times 10^{-5}$ & $2.2585 \times 10^{-5}$ \\
9 & 6 & 0.000166904 & 0.000208757 \\
9 & 7 & 0.000243874 & -0.000117568 \\
9 & 8 & -0.00026601 & -0.000206909 \\
9 & 9 & -0.000681636 & 0.000388259 \\
10 & 0 & $3.8741 \times 10^{-5}$ & 0 \\
10 & 1 & 0.000162276 & $1.95518 \times 10^{-5}$ \\
10 & 2 & $-3.39526 \times 10^{-5}$ & $7.28263 \times 10^{-6}$ \\
10 & 3 & -0.000144807 & $-6.0498 \times 10^{-5}$ \\
10 & 4 & $-2.35412 \times 10^{-5}$ & $-8.49923 \times 10^{-6}$ \\
10 & 5 & 0.000116467 & 0.000105469 \\
10 & 6 & $9.94341 \times 10^{-5}$ & $-1.74278 \times 10^{-6}$ \\
10 & 7 & $-8.93117 \times 10^{-5}$ & -0.000151414 \\
10 & 8 & -0.000153653 & $3.23242 \times 10^{-5}$ \\
10 & 9 & $7.56925 \times 10^{-5}$ & 0.000180907 \\
10 & 10 & 0.000105512 & -0.000131908 \\
\hline \hline & & &
\end{tabular}

Table 3. Direction Cosines

\begin{tabular}{c|ccc}
\hline \hline & $\hat{\mathbf{b}}_{1}$ & $\hat{\mathbf{b}}_{2}$ & $\hat{\mathbf{b}}_{3}$ \\
\hline$\hat{\mathbf{e}}_{1}$ & $\cos \theta$ & $-\sin \theta$ & 0 \\
$\hat{\mathbf{e}}_{2}$ & $\cos i \sin \theta$ & $\cos i \cos \theta$ & $-\sin i$ \\
$\hat{\mathbf{e}}_{3}$ & $\sin i \sin \theta$ & $\sin i \cos \theta$ & $\cos i$ \\
\hline \hline
\end{tabular}

11 of 15

American Institute of Aeronautics and Astronautics 

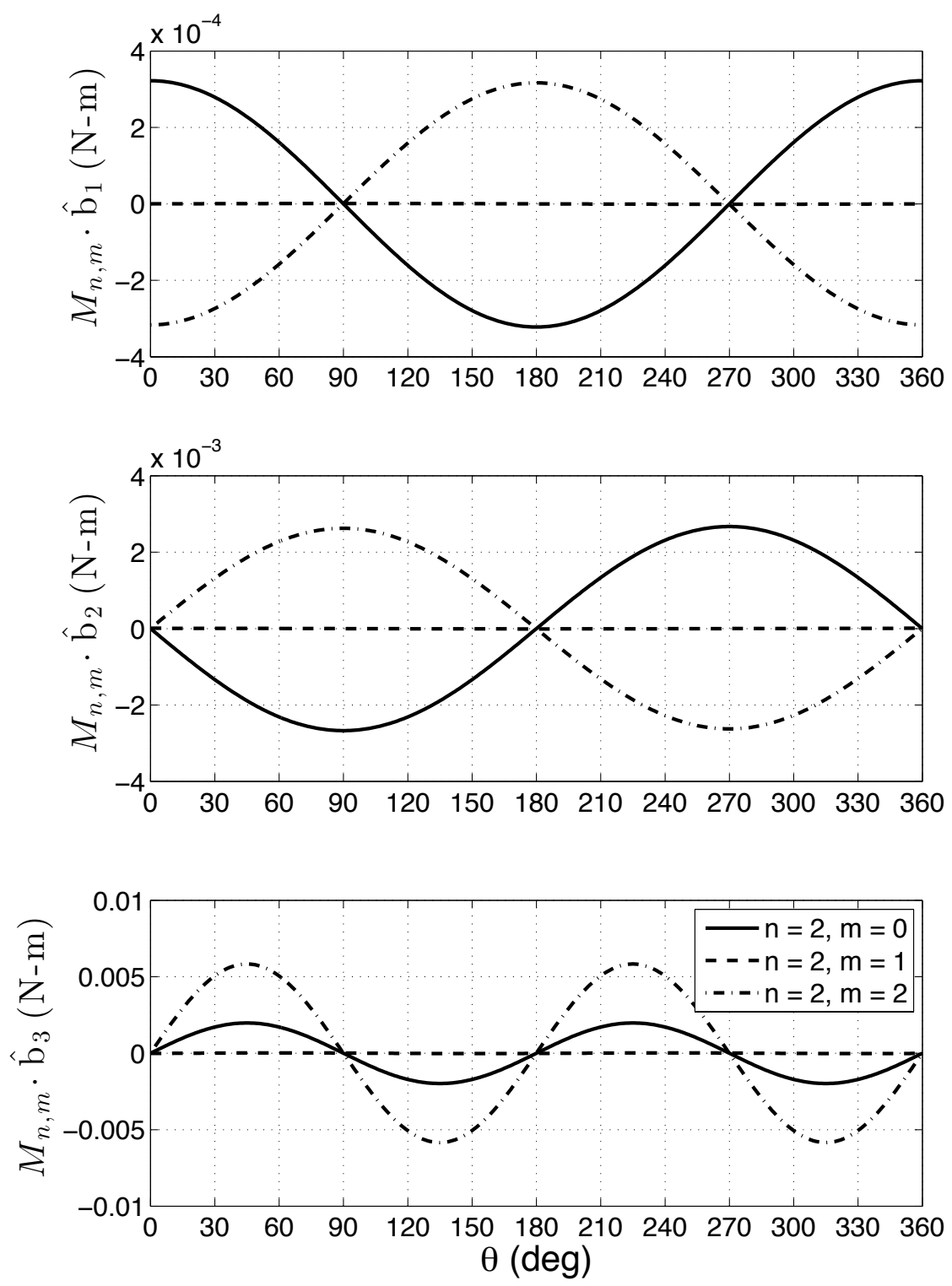

Figure 4. Contributions of degree 2 to gravitational moment. 

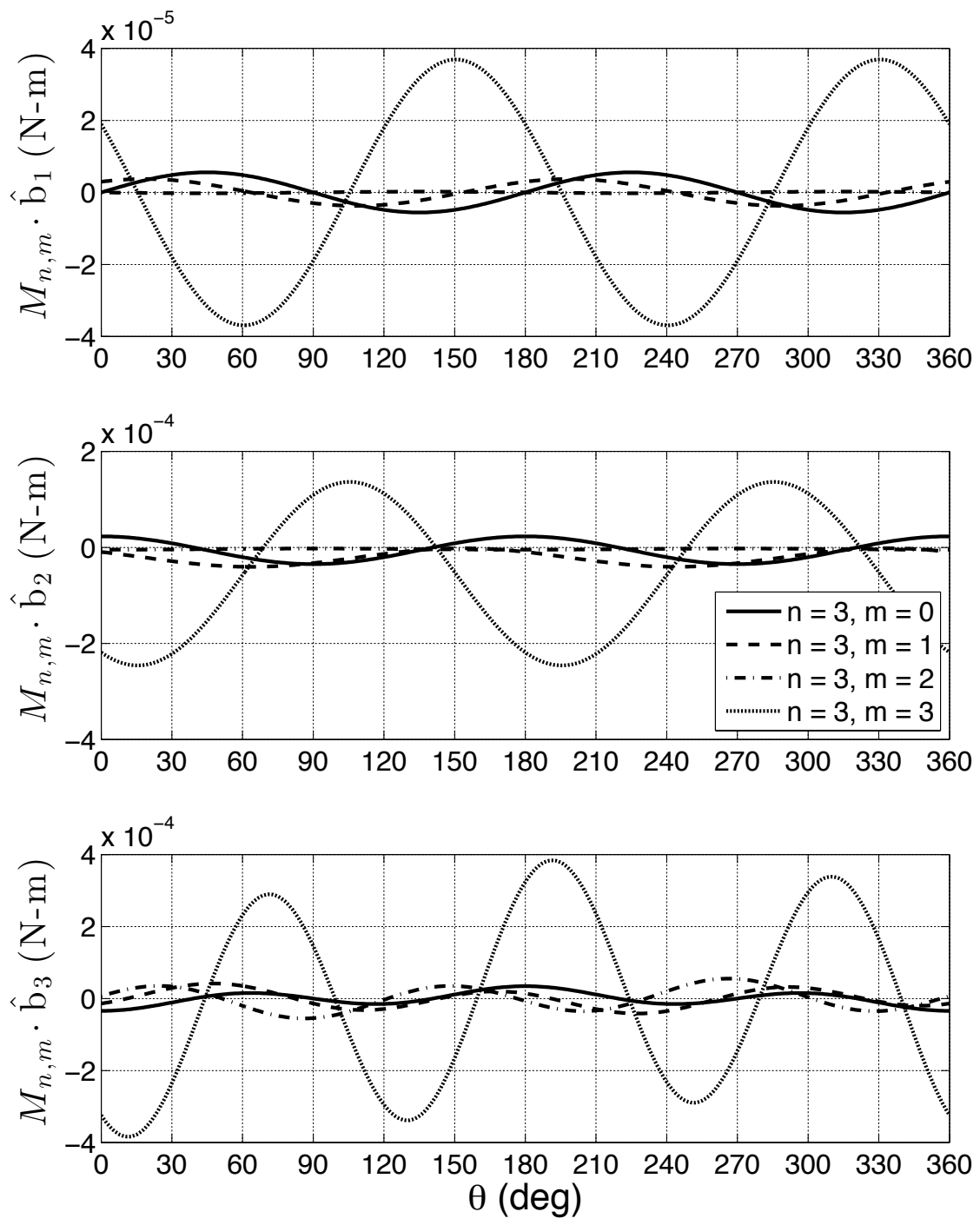

Figure 5. Contributions of degree 3 to gravitational moment. 

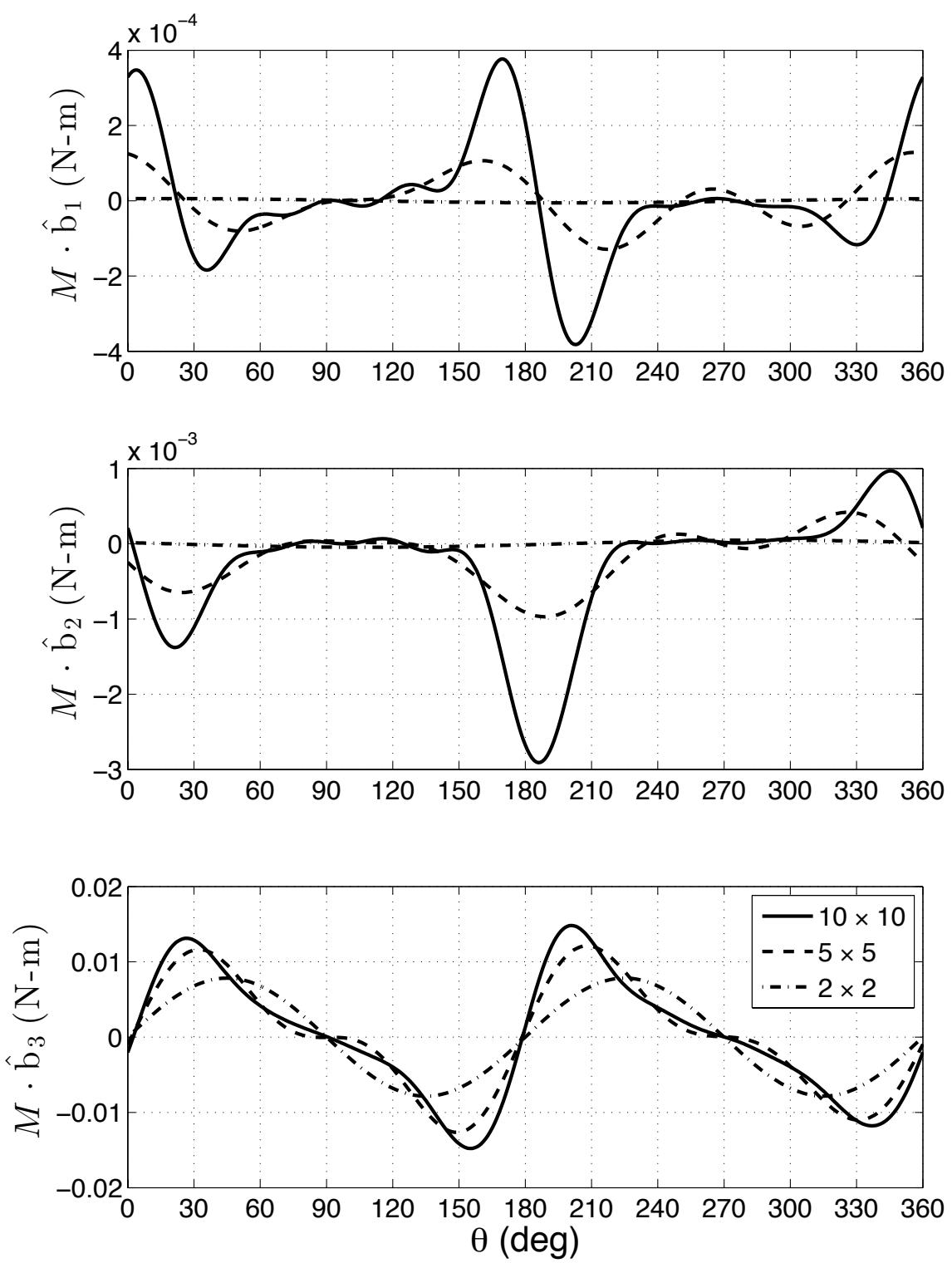

Figure 6. Gravitational moment calculated with $2 \times 2,5 \times 5$, and $10 \times 10$ gravity models. 


\section{Conclusions}

The gravitational potential of an astronomical body is commonly represented as a series of spherical harmonics. The paper presents a vector-dyadic expression for the contribution of a generic spherical harmonic to the moment of gravitational forces about the mass center of a small body, exerted by an astronomical body. Expressions related to the contribution of a spherical harmonic to gravitational gradient can be employed to construct a state propagation matrix and a state transition matrix, which are useful for trajectory targeting and Kalman filtering. The expressions presented here in connection with gravitational gradient can also be used in numerical simulations involving measurements obtained with a gradiometer in low-Earth orbit. Contributions of spherical harmonics to gravitational moment about the mass center of a spacecraft are evaluated numerically for a series of positions near the asteroid 216 Kleopatra. Results obtained when the gravity model is truncated to degree and order $N=10$ are noticeably different from results obtained with $N=5$ and $N=2$.

\section{Acknowledgments}

The author would like to express his sincere gratitude to Rachit Bhatia and Prof. David Geller for pointing out the literature concerned with orbit determination based on gradiometer measurements, to Prof. Othon Winter for discussing the parameters used in producing the spherical harmonic coefficients for Kleopatra, and to Haijun Shen for assistance in manipulating the shape model of Kleopatra.

\section{References}

\footnotetext{
${ }^{1}$ Werner, R. A., and Scheeres, D. J., "Exterior Gravitation of a Polyhedron Derived and Compared with Harmonic and Mascon Gravitation Representations of Asteroid 4769 Castalia," Celestial Mechanics and Dynamical Astronomy, Vol. 65, No. 3, 1997, pp. 313-344.

${ }^{2}$ Roithmayr, C. M., "Gravitational Moment Exerted on a Small Body by an Oblate Body," Journal of Guidance, Control, and Dynamics, Vol. 12, No. 3, 1989, pp. 441-444.

${ }^{3}$ Roithmayr, C. M., "Contribution of Zonal Harmonics to Gravitational Moment," Journal of Guidance, Control, and Dynamics, Vol. 14, No. 1, 1991, pp. 210-214.

${ }^{4}$ Battin, R. H., An Introduction to The Mathematics and Methods of Astrodynamics, AIAA, New York, 1987, Chap. 9.

${ }^{5}$ Tapley, B. D., Schutz, B. E., and Born, G. H., Statistical Orbit Determination, Elsevier Academic Press, Amsterdam, 2004, Chap. 4.

${ }^{6}$ Sun, X., Chen, P., Macabiau, C., and Han, C., "Low-Earth Orbit Determination from Gravity Gradient Measurements," Acta Astronautica, Vol. 123, 2016, pp. 350-362.

${ }^{7}$ Sun, X., Chen, P., Macabiau, C., and Han, C., "Autonomous Orbit Determination via Kalman Filtering of Gravity Gradients," IEEE Transactions on Aerospace and Electronic Systems, Vol. 52, No. 5, 2016, pp. 2436-2451.

${ }^{8}$ Lear, W. M., The Gravitational Acceleration Equations, NASA Johnson Space Center, 86-FM-15, April, 1986.

${ }^{9}$ Takahashi, Y., Busch, M. W., and Scheeres, D. J., "Spin State and Moment of Inertia Characterization of 4179 Toutatis," The Astronomical Journal, Vol. 146, No. 4, 2013.

${ }^{10}$ Mueller, A. C., A Fast Recursive Algorithm For Calculating The Forces Due To The Geopotential, NASA Johnson Space Center Internal Note No. 75-FM-42, June 9, 1975.

${ }^{11}$ Lundberg, J. B., and Schutz, B. E., "Recursion Formulas of Legendre Functions for Use with Nonsingular Geopotential Models," Journal of Guidance, Control, and Dynamics, Vol. 11, No. 1, 1988, pp. 32-38.

${ }^{12}$ Roithmayr, C. M., Contributions of Spherical Harmonics to Magnetic and Gravitational Fields, NASA TM-2004-213007, March, 2004.

${ }^{13}$ Gottlieb, R. G., Fast Gravity, Gravity Partials, Normalized Gravity, Gravity Gradient Torque and Magnetic Field: Derivation, Code, and Data, NASA CR-188243, February, 1993.

${ }^{14}$ Kane, T. R., Likins, P. W., and Levinson, D. A., Spacecraft Dynamics, McGraw-Hill, New York, 1983, Chap. 2.

${ }^{15}$ Pines, S., "Uniform Representation of the Gravitational Potential and its Derivatives," AIAA Journal, Vol. 11, No. 11, 1973, pp. 1508-1511.

${ }^{16}$ Ostro, S. J., Hudson, R. S., Nolan, M. C., Margot, J.-L., Scheeres, D. J., Campbell, D. B., Magri, C., Giorgini, J. D., and Yeomans, D. K., "Radar Observations of Asteroid 216 Kleopatra," Science, Vol. 288, No. 5467, 2000, pp. 836-839.

${ }^{17}$ Chanut, T. G. G., Winter, O. C., Amarante, A., and Araújo, N. C. S., "3D Plausible Orbital Stability Close to Asteroid (216) Kleopatra," Monthly Notices of the Royal Astronomical Society, Vol. 452, No. 2, 2015, pp. 1316-1327.

${ }^{18}$ Werner, R. A., "Spherical Harmonics Coefficients for the Potential of a Constant-Density Polyhedron," Computers $\&$ Geosciences, Vol. 23, No. 10, 1997, pp. 1071-1077.
} 